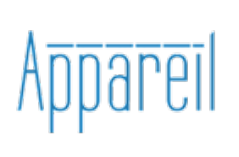

Appareil

$6 \mid 2010$

Philosophie et cinéma

\title{
La fragmentation, ce monstrueux
}

Étude sur le décloisonnement disciplinaire dans l'œuvre de Lynch

Marion Delage de Luget

\section{CpenEdition}

Journals

Édition électronique

URL : http://journals.openedition.org/appareil/420

DOI : 10.4000/appareil.420

ISSN : 2101-0714

Éditeur

MSH Paris Nord

Référence électronique

Marion Delage de Luget, "La fragmentation, ce monstrueux », Appareil [En ligne], 6 | 2010, mis en ligne le 15 février 2010, consulté le 30 juillet 2020. URL : http://journals.openedition.org/appareil/420 ; DOI : https://doi.org/10.4000/appareil.420

Ce document a été généré automatiquement le 30 juillet 2020

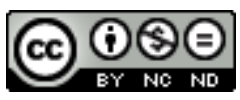

Appareil est mis à disposition selon les termes de la Licence Creative Commons Attribution - Pas d'Utilisation Commerciale - Pas de Modification 4.0 International. 


\title{
La fragmentation, ce monstrueux
}

\author{
Étude sur le décloisonnement disciplinaire dans l'œuvre de Lynch
}

\section{Marion Delage de Luget}

1 Que le kit présuppose l'éventualité d'un montage, comme le dit Michel Chion ${ }^{1}$, signifie que le fragment, la log, réitère - en continu - l'indexation d'une totalité. «[...] le plan lui-même, [dit-il,] comme fragment intermédiaire reliant deux autres plans, est fragment d'un continuum auquel il renvoie." Soit, ici à propos de la pratique cinématographique de Lynch : il ne peut être question de coupure sans qu'il soit aussi, et aussitôt, question d'enchaînement.

2 Pas seulement en ce que le cut, par section, appelle ou suppose l'adjonction; mais plutôt, comme le proposent Philippe Lacoue-Labarthe et Jean-Luc Nancy, en ce sens que tout fragment est projet: «[...] le fragment-projet ne vaut pas comme programme ou prospective, mais comme projection immédiate de ce que pourtant il inachève ${ }^{2}$. C'est $^{2}$ ce que le fameux fragment 206 de l'Athenaum énonce : «Pareil à une petite œuvre d'art, un fragment doit être totalement détaché du monde environnant, et clos sur lui-même comme un hérisson ${ }^{3}$.»

3 Nancy et Lacoue-Labarthe épinglent dans ce fragment le «à la fois » qui articule ce qu'ils nomment la logique du hérisson. D'abord, le fragment est compris comme détaché, isolé, et désignant ou recouvrant une totalité - «Son devoir-être, sinon son être [...], est bien formé par l'intégrité et l'intégralité de l'individualité organique ${ }^{4}$.» Pourtant, ce fragment 206 ouvre par un comparatif : cette complétude n'est là, en somme - littéralement en somme -, qu'en regard de l'œuvre d'art. Autrement dit, si le fragment, bien qu'il soit une totalité, n'est pas l'œuvre, il demeure pourtant « pareil à », son individualité propre toujours tributaire du rapport définitoire, d'avec cette « petite œuvre d'art ».

4 C'est cette même dynamique qui désigne le mouvement à l'œuvre dans la notion d'interdisciplinarité - pour peu que l'on s'accorde à justifier par l'étymologie le choix de ce préfixe, inter, par exclusion des acceptions plus permissives que l'usage commun lui a peu à peu substitué, en redoublant son sens premier par les définitions approximatives de termes comme multidisciplinaire ou pluridisciplinaire. Termes approchants, mais non synonymes, comme le rappelle Patrice Loubier : «Si les préfixes 
"multi" et "pluri" signifient "beaucoup, grand nombre", "plusieurs", le préfixe "inter", lui, désigne tout autant la relation qui unit ces différents éléments que l'espacement même qui les sépare ${ }^{5}$." D'un côté, donc, une dimension strictement quantitative, mais de l'autre un espace médiateur. Un espace qui se situe entre les disciplines, à la fois les reliant et les indexant comme autant d'objets partiels; un espace au sein duquel à la tradition forclose d'un médium donné répondra la relation de ce médium à d'autres. L'interdisciplinarité, dans l'analyse du travail de Lynch, c'est ce concept par lequel l'œuvre trouve sens quant aux relations complexes qui existent entre spécialisation (fragmentation) et généralisation (principe d'unité).

Pour le dire autrement, et soutenir la parenté avec l'idée de fragment-projet, c'est à peu de chose près ce que Nancy et Lacoue-Labarthe résument par cette formule :

L'individualité fragmentaire est avant tout celle de la multiplicité qui est inhérente au genre $[. . .]^{6}$.

Écrire en fragment, disent-ils encore, juste après, c'est écrire en fragments. Mais ce pluriel est le mode spécifique par lequel le fragment vise, indique et d'une certaine façon pose le singulier de la totalité7.

6 Aussi, proposer une analyse interdisciplinaire de l'œuvre de David Lynch, ce ne sera pas seulement mettre en rapport des métiers, ou supposer une somme. Si l'on conçoit chaque pratique disciplinaire comme un fragment du corpus interdisciplinaire, ce corps - ou grand œuvre -, ne sera pas pour autant la totalité fragmentaire, puisque cette totalité singulière est en quelque sorte atopique : «[...] elle est simultanément dans le tout et dans chaque partie ${ }^{8} "$, disent Lacoue-Labarthe et Nancy. De la même façon que l'inter, compris comme tissu conjonctif entre les pratiques, se joue simultanément dans les spécificités inhérentes à chaque discipline, comme clôtures, et hors d'elles. Postuler l'interdisciplinarité, c'est gager que, dans cette simultanéité, le tout et la somme des parties ne se recouvrent jamais vraiment. Sinon, multi, pluri, auraient suffit à quantifier. Nancy et Lacoue-Labarthe concluent sur ce point en parlant de co-présence pour caractériser le mode d'être du fragment, des fragments, à la totalité singulière: «Les fragments sont au fragment ses définitions, et c'est ce qui installe sa totalité comme pluralité, et son achèvement comme inachèvement de son infinité9.»

7 Sans doute, alors, dans cette analyse interdisciplinaire, les notions de médium ou de discipline, de partie, et de totalité, devront-elles céder la place à une typologie fondée sur les opérations selon lesquelles elles peuvent interagir. Parce que l'interdisciplinarité suppose un questionnement des frontières disciplinaires, c'est-àdire des mécanismes de liaison et d'exclusion, de différence et d'identité, structurant tant la discipline, les disciplines regroupées sous le vocable commun d'" art », que ce qui, hors d'elle - au singulier-, hors d'elles - au pluriel-, peut devenir inter, peut s'affirmer milieu. Ce milieu qui, si l'on poursuit dans l'idée d'une discipline comme fragment, véhicule, pour Nancy et Lacoue-Labarthe, cette double signification de l'exergue: c'est ce qui s'inscrit hors de l'œuvre, et c'est ce qui l'accomplit. «Le fragment figure - nous disent-ils - mais figurer, bilden et gestalten, c'est ici œuvrer, et présenter, darstellen - le hors-d'œuvre essentiel à l'œuvre, plus essentiel à l'œuvre que l'œuvre elle-même ${ }^{10}$.» D'une certaine façon, donc, l'œuvre, pour eux, est absente des œuvres - c'est ce que la fragmentation indexe. Ce qu'ils qualifient d'œuvre véritable, l'œuvre absolue, est bien plus "[...] cette "vie de l'esprit" en qui "vivent tous les individus" [...]». Et puisque le fragment reprend la configuration d'un individu 
organique, c'est donc dans la forme de la fragmentation, par cette opération les donnant comme totalité plurielle, que l'œuvre s'accomplit.

8 L'inter, comme hors-d'œuvre, prend également forme entre tous ces champs connexes qui le déterminent du dehors. La pensée esthétique classique, tout comme l'histoire de l'art, jusqu'il y a peu, astreignaient les pratiques artistiques à un découpage par médium. Distinguer ainsi par matériaux et méthodes, sectoriser la production d'œuvres en savoir-faire, voilà pour socle du modernisme de Greenberg: la dynamique d'autonomisation de l'art qu'il envisage n'étant rien moins qu'une division par spécialisation. Un morcellement, donc, pas une fragmentation. Pourtant, dès le $\mathrm{xIX}^{\mathrm{e}}$ siècle, et au début $\mathrm{du} \mathrm{xx}^{\mathrm{e}}$, l'histoire de l'art s'est enrichie de sources archéologiques, philosophiques, philologiques, de la psychologie de la perception, de la biologie, de la sociologie et du marxisme. Des apports méthodologiques qui conduiront à ce que les années 70 entérineront sous le nom de new art history, une nouvelle approche critique de l'art, encore révisée dans les années 1980 par cette idée, à l'époque juste émergente avec les cultural studies, d'un nécessaire dialogue transversal entre les diverses branches de savoir. Le processus moderne proposait une segmentation de la connaissance en disciplines toujours plus spécialisées. L'interdisciplinarité, opération post-moderne, déjouera cette dynamique de parcellisation du savoir. Parce qu'elle permet, nous dit Patrice Loubier, «[...] la mise en perspective critique des méthodes et présupposés d'une branche par une autre (en d'autres termes, la mise au jour de la relativité inhérente à tout discipline) ${ }^{11}$. »

C'est d'ailleurs pourquoi Nancy et Lacoue-Labarthe notent la parfaite justesse avec laquelle les romantiques allemands ont mis en œuvre cette forme fragmentaire pour redéfinir le genre littéraire; parce que Schlegel et les autres savaient le caractère relatif du fragment, cette dialectique de l'achèvement et de l'inachèvement qui lui est propre, tant dans sa forme singulière que comme totalité plurielle. C'était déjà entendu dans leur définition : « Le fragment sur le fragment-hérisson est tel un hérisson dans sa proposition même, par laquelle il énonce que le hérisson n'est pas là ${ }^{12}$. ^ À quoi LacoueLabarthe et Nancy ajoutent cette image frappante d'un couronne de fragments dessinant en son centre un vide, à la fois hors-d'œuvre, donc, mais reprenant aussi très exactement les contours de l'œuvre. Ils précisent: «L'œuvre en ce sens est absente des œuvres - et la fragmentation est toujours aussi le signe de cette absence ${ }^{13}$.»

C'est ainsi, précisément, dans la relativité de méthodes et de formes qui sous-tend l'interdisciplinarité, qu'une perspective critique sera envisageable - et qu'elle pourra prétendre dépasser les clivages disciplinaires (tant pratiques que théoriques) sans pour autant présager la perte de la spécificité matérielle de l'œuvre. Si le fragment permet l'énoncé « l'œuvre est absente des œuvres ", c'est parce qu'il est signe de cette absence. Soit, justement, parce que la configuration fragmentaire - la totalité plurielle de la couronne - désigne la Physionomie absolue de l'œuvre d'art. Nancy et Lacoue-labarthe disent : «Au-delà ou en-deçà de l'œuvre il en propose l'opérativité même ${ }^{14}$. » Gageons que la multiplication des pratiques interdisciplinaires est signe d'un questionnement comparable : nourrit du dehors par les pensées poststructuralistes et postcolonialistes, par les recherches féministes, les genders et queer studies, l'art contemporain déconstruit lui aussi cette définition de l'identité comme englobante, homogène et unifiée.

11 L'identité n'est plus cette entité cohérente et permanente, autosuffisante et reconnaissable. Ce qui ne revient pas à dire que l'identité est portée absente, bien au 
contraire : elle se diversifie et se complexifie. Pour le dire autrement, et revenir au fragment, l'art contemporain a peut-être ceci de spécifique dans la mise en œuvre de pratiques interdisciplinaires qu'il y trouve le lieu et signe de son opérativité même. L'histoire de l'art et des techniques montre nombre d'exemples antérieurs de rencontres disciplinaires au sein d'un même Æuvre. Mais cette interdisciplinarité postmoderne, qui trouve source dans la distraction et la tabula rasa de l'internationale dadaïste, dans l'effrangement des espaces et des techniques des collages cubistes, dans ce geste expressionniste ou toujours, sur la technique, l'intention prime, dans cette attention aux formes artistiques extra-européennes, dans le ready-made, dans l'investissement de peintres, de sculpteurs, de poètes, d'architectes, pour l'émergence des arts graphiques et appliqués, dans tous ces actes par lesquels les avant-gardes déconstruiront la visée académique et l'aune d'un regard ethnocentré, cette interdisciplinarité-là introduit des possibles par différence ou contradiction interne qui permettent de repenser entièrement l'identité de l'œuvre, c'est-à-dire aussi les limites de chaque discipline, et les attendus et présupposés de leur lecture croisée.

On pourrait croire ce projet ambivalent. Parce que, d'un côté, il ne s'agit pas d'hypostasier les spécificités matérielles des pratiques artistiques - on sait les limites de l'essentialisme greenbergien -, mais il ne s'agit pas non plus de les nier, puisque ce sont ces caractéristiques intrinsèques, comme limites définitoires de la fragmentation de l'art en pratiques, qui produisent l'inter. Penser cette propriété équivoque du fragment comme dialectique de l'achèvement et de l'inachèvement pose en fait la question du point de vue critique. Si l'on suit Nancy et Lacoue-Labarthe, ce caractère ambigu du fragment pourrait être saisi de telle façon qu'il «[...] reviendrait à concentrer ou précipiter sur un point le processus par lequel le discours philosophique, chez Hegel [...], peut désigner son propre inachèvement, le maîtriser et le faire passer dans l'élément de la "pure pensée", qui est son achèvement ${ }^{15}$. » Un mouvement qui suppose donc une solution hors champ: le fragment, la fragmentation, trouveraient leur achèvement hors d'eux et hors de l'œuvre, dans la pure pensée. Et, conséquemment, cela reviendrait à présenter le hérisson non plus comme un individu organique, comme unité et intégralité, mais comme une collection de membres épars, ce morcellement de l'unheimlich propre à la perte de l'individualité. Pourtant, insistent les auteurs, la fragmentation n'est pas une dissémination, au sens précis qu'en donne Derrida: ce n'est pas cette " [...] dispersion stérile de la semence et du sémique en général, c'est-àdire du signe et du sens ${ }^{16}{ }^{\prime}$, qui ne reprendrait corps qu'en une pensée qui lui serait extérieure. Tout au contraire, c'est le système même de cette fragmentation qui est lieu de production et qui produit. Nancy et Lacoue-Labarthe parlent alors de "production originelle », pour appuyer encore que cette dispersion est de celle " [...] qui convient à l'ensemencement et aux futures moissons. Le genre du fragment est le genre de la génération ${ }^{17}$.»

Ainsi, le genre du fragment génère œuvre et hors-d'œuvre, signe et sens, mais aussi une critique esthétique toute particulière : dans la fragmentation, selon Nancy et LacoueLabarthe, «La théorie [...] est consubstantielle et coextensive à l'opération ${ }^{18}$.» Cette ouverture du fragment 117 du Lycée le rappelle : «La poésie ne peut être critiquée que par la poésie ${ }^{19}$. $) E t$, dans un premier temps, très simplement y voir la nécessité d'ouvrir l'œuvre interdisciplinaire à une analyse recréant elle aussi un tissu conjonctif; donc, comme annoncé, une pensée puisant dans des champs théoriques, sémiotiques, et méthodologiques plus larges, parce qu'il faut d'une certaine manière que l'organon, 
l'instrument de la pensée, redouble le motif de la couronne, pour que la critique ne manque pas son objet et son propos : interdisciplinaires.

Que ce concept de fragmentation serve l'exemple critique appelle encore une précision : ajoutons que, comme le fragment, cette critique, nécessairement, "manque" l'œuvre. La pensée critique interdisciplinaire, comme la fragmentation, est signe de l'absence de l'œuvre, puisque si elle fait écho à son opérativité, tout comme elle, elle se borne alors à en souligner les contours, la béance. Très simplement, parler de l'œuvre comme on parle du hérisson; et savoir déjà qu'être à en toucher les bords c'est y être extérieur, c'est accepter de la perdre. Il n'y a que la poésie qui puisse critiquer la poésie, disent les romantiques, parce que la pure pensée n'inclut plus ce manque essentiel à la génération; parce qu'elle est, au contraire, tentative de maîtrise de la perte - ou sa simple obstruction, c'est de même.

Alors que, justement, tout prend place dans cet écart par lequel la fragmentation peut opérer. Tout se joue en ce que le fragment est du genre de la génération. Le genre, pour les romantiques, est "plus qu'un genre ${ }^{20} »$, c'est un individu. Un Hérisson, un organisme. Un Tout qui, comme l'organique, s'engendre du fragment et par le fragment. Et c'est aussi en cela qu' " aucune théorie ne peut l'épuiser ${ }^{21}$ ", parce que, rappellent Lacoue-Labarthe et Nancy, il est probable que le genre soit, par essence, indéfini et illimité :

Sans doute le genre est-il le produit, achevé, différencié, identifiable d'un engendrement ou d'une génération; et même en allemand, où la filiation étymologique du mot est toute différente, Gattung n'est pas sans rapport avec l'assemblage en général, l'union - voire le mariage. Mais le procès de la génération, justement, ou le procès de l'assemblage suppose - c'est l'évidence interpénétration et confusion. C'est-à-dire mélange [... ${ }^{22}$.

C'est là, c'est exactement là que l'œuvre interdisciplinaire rompt avec l'académisme et le modernisme. Il n'y a pas de genre interdisciplinaire dans le sens d'une production achevée, différenciée, et identifiable, en ce sens que la pratique, l'œuvre ou la pensée interdisciplinaire ne s'inscrivent pas dans un processus de continuité, ni dans une logique d'engendrement comme reproduction du même. L'esthétique classique, en posant les disciplines en termes de hiérarchie, assurait en fait la définition de chacune selon une place fixe dans la systématique. Comme un biologiste subdivise une famille en genres, l'art, la pensée sur l'art, épousaient la structure d'une arborescence établie sur de rigoureux principes d'ordre normatifs. Le rêve moderniste n'est pas loin: une autonomisation de chaque discipline dans la conformité exclusive aux conditions matérielles de sa génération, soit encore cette même idée de la synchronicité d'un monde homogène. L'interdisciplinaire n'est pas ce genre, dans son acception biologique. Ou alors, si on l'y rapporte, faut-il l'entendre comme ce qui, dans la continuité, peut être le dissemblable. L'inter bouscule les catégories en vigueur ; en cela, il est monstrueux. Le schème de la reproduction de l'espèce est finalisé par la loi de la ressemblance. Cela veut dire que c'est en vertu de cette idée de ressemblance que seront instaurés les critères de l'espèce et les principes de classification. Deux développements, à partir de ce point: sur la valeur opératoire des systèmes taxinomiques, puis sur la question de l'identité genrée.

Dans les conceptions classiques et modernistes, une fois cernées et établies, les propriétés alors permanentes de l'œuvre prenaient valeur de lois. C'est-à-dire qu'elles permettaient une systématique par différenciation. Le monstre étant alors ce qui se trouve hors sens ou hors limites, autant l'anomal - que ne recouvre aucune catégorie - 
que ce qui transgresse les espacements constitutifs des critères spécifiques. Mary Douglas a pointé la mécanique de ces rites de séparation. Pour en donner l'élémentaire, selon elle, les notions de souillure, de pollution, de tabou, ne prennent sens que dans le contexte de rituels définissants des interdits, soit des marges, des limites, des frontières. Autrement dit, la notion de pollution est relative; elle dépend toujours des interdits rituels qui, en édifiant la structure de la pensée d'une culture, vont dans le même mouvement donner, pour cette même culture, une définition de la souillure par la négative. «La souillure n'est jamais un phénomène isolé. - dit Mary Douglas - Elle n'existe que par rapport à l'ordonnance systématique des idées ${ }^{23}$. » Là aussi, il faut donc s'attacher à comprendre le système. Douglas illustre sa thèse en proposant une relecture des extraits du Lévitique et du Deutéronome désignant les abominations et les prescriptions alimentaires. Il est dit, dans le Deutéronome :

3. Tu ne mangeras rien d'abominable ! [...] 6. toute bête qui a le pied onglé, l'ongle fendu en deux, et qui fait partie des ruminants, vous en mangerez. 7. Mais vous ne mangerez pas [...] le chameau, le lièvre, le daman, car ils ruminent, mais ils n'ont pas l'ongle fendu: ils seront impurs pour vous. 8. Ni le porc, car il a bien l'ongle fendu, mais il ne rumine pas : il sera impur pour vous. Vous ne mangerez pas de leur chair et vous ne toucherez pas à leur cadavre ${ }^{24}$.

Les interprétations historiques de ce texte appuient généralement l'idée d'une règle disciplinaire pour en expliquer l'arbitraire; ou prétendent l'allégorie des vertus et des vices. L'un comme l'autre, c'est se fourvoyer, selon Douglas, pour qui la clef de lecture réside dans une approche critique de la notion de sainteté. Une notion qui, rappelle-telle, véhicule un sens restrictif : la racine latine du terme, sacer, tout comme sa racine hébraïque, étant fondée directement sur l'idée de séparation. Le rituel scinde donc le monde entre observance et désobéissance, les permissions édictant l'idéal, et les interdictions promettant maints dangers pour l'insoumis. En art, pareillement, l'inter est cet indiscipliné : il est l'abominable qui déroge à la logique du concept, cette présence que la classification veut rejeter hors propos parce qu'elle invalide l'exclusif de la structure disciplinaire.

19 Mais la notion de sainteté implique encore davantage, pour Douglas : "S'il est admis que la racine de ce mot signifie : état de séparation, la notion de sainteté comprend aussi celle de totalité, de plénitude ${ }^{25}$. » Et de rappeler que les allusions à la perfection physique abondent dans le Lévitique. Point de règle arbitraire, donc, ni d'allégorie: c'est l'idée de complétude qui guide toute taxinomie. "Être saint, nous dit mary Douglas, c'est distinguer soigneusement les différentes catégories de la création, c'est élaborer des définitions justes, c'est être capable de discrimination et d'ordre ${ }^{26}$. . Les $^{\prime}$ rites exigent une différenciation, puis l'assurent, par obédience. D'une façon très similaire, tout classement distinguant l'art en disciplines vise avant tout à préserver un ordre sur lequel est fondé la symbolique culturelle du groupe - autrement dit, la norme, dans son arbitraire. Quant aux abominations alimentaires auxquelles il était tout d'abord fait allusion, la solution discernant sacré et souillure est donc donnée :

Être saint, dit encore Douglas, c'est être entier, être un; la sainteté, c'est l'unité,

l'intégrité, la perfection de l'individu et de ses semblables. Les prescriptions

alimentaires se contentent de développer la métaphore dans le même sens ${ }^{27}$.

Face au concept de discipline, face à aux classifications diverses, c'est effectivement l'idée de conformation aux distinctions premières, quelles qu'elles soient, qui importe. Conformation et conformité : il ne saurait y avoir doute, ni dans la filiation, ni entre les différents objets. Le système taxinomiste maintient les séparations définitoires, et ainsi 
non seulement garantit l'intégrité de chacun mais, par là, assure sa pérennité dans la production itérative du même. Douglas revient aussi sur cette subtilité de traduction du terme "perversion" dans le Lévitique : le texte énonce «Et à aucune bête tu ne donneras ton épanchement pour en devenir impur et une femme ne se donnera pas à une bête pour s'accoupler avec elle: c'est là une perversion ${ }^{28}$." Selon elle, ce mot, perversion, est « [...] une erreur significative du traducteur. L'original, en hébreu, est thebel, qui signifie "mélange", ou confusion ${ }^{29}$." À l'impératif de la loi de la ressemblance, la perfection de l'individu et de ses semblables, s'ajoute donc l'impossible reconnaissance du trans-genre : entre autres préceptes, le Lévitique entérine : pas de croisement entre deux espèces de bétail, pas de culture mêlant deux types de semences en un même sol, pas de tissage, non plus, réunissant des fils de fabriques différentes.

21 Le monstre est monstre parce qu'il transgresse les écarts constitutifs de l'un à l'autre. En ce sens, il est déjà monstrueux de dire que le hérisson est pareil à ; il devrait être ou n'être pas cet autre. Le à la fois du fragment, de l'inter, c'est cette totalité plurielle qui dément les critères du genre. Ou plutôt, c'est cette même opération qui, à l'idée biologique d'un genre invariable et occupant une place fixe dans la systématique, substitue celle d'un genre logique, un concept en englobant d'autres, unité par union, proche de l'assemblage du gattung. Ainsi la fragmentation, l'interdisciplinarité, constitue véritablement ce point d'incohérence où s'amorce la rupture d'un ordre; parce qu'elle valide une nouvelle structure, basée sur la confusion, une structure qui n'est plus que marges, un système opérant dans les rencontres, avec tout ce qu'il peut y avoir d'inconvenant à ne plus séparer ce qui doit l'être.

Deux alternatives, alors. Puisque l'anomal, le monstrueux, le hors norme, induisent la possibilité d'une refonte complète du système, on peut par exemple choisir de trouver de nouveaux principes de catégorisation. Somme toute, il suffit d'étiqueter la dérogation pour qu'elle cesse de l'être. C'est ce que tente la tératologie, cette science des monstres apparue au XIX ${ }^{\mathrm{e}}$ siècle, avec Étienne et Isidore Geoffroy Saint-Hilaire, qui trouvera ensuite écho dans la tératogenèse expérimentale, puis, jusqu'à aujourd'hui, dans la biologie du développement. Une pensée à propos de laquelle Jean Gayon souligne qu'«Elle n'a cessé de ronger le concept de monstre en lui conférant une banalité biologique ${ }^{30}$. $»$ Effectivement, la tératologie a procédé à une classification des formes monstrueuses. Jean Gayon commente :

Étienne Geoffroy Saint-Hilaire en faisait des formes d'organisation achevées, qu'il regroupait en des taxons aussi distincts entre eux que le sont les espèces, les genres, les familles d'organismes communément construites par les naturalistes. Par ce premier pas, la tératologie dissolvait la vieille idée de jeu ou de caprice de la nature : les monstres devenaient des formes réglées, dont les propriétés constantes avaient valeur de lois de la nature ${ }^{31}$.

Le monstre est ainsi normalisé. Jusque dans ce paradigme de la tératologie que Goldschmidt a développé par la rhétorique du «monstre prometteur». La formule désigne ces cas ou la formation d'une espèce nouvelle résulte d'une unique mutation monstrueuse. Pour fonder son propos, Goldschmidt s'appuie lui-même sur les recherches de William Bateson concernant les mutants homéotiques: Jean Gayon explique :

[...] l'homeosis consiste en ce qu'un organe est remplacé par un autre dans un organisme comportant des parties répétées [...]. Bateson avait ainsi décrit des insectes dans lesquelles une paire d'antennes se trouvait transformée en une paire de pattes, une paire de pattes en une paire d'ailes, un œil en antenne ${ }^{32}$. 
S'appuyant sur ces données, Goldschmidt fera ce constat: certaines formes tératologiques paraissent très semblables aux standards d'une espèce apparentées. Il prend exemple d'une mouche, la Termitoxenia, dont la seconde paire d'aile, atrophiée, rappelle la morphologie des haltères de certains diptères ayant subit une mutation homéotique. Jean Gayon conclue :

les ressemblances entre anomalies dans une espèce et forme normale dans une autre suggérait que l'état normal de telle espèce avait son origine dans une mutation tératogène ayant affecté un ancêtre ${ }^{33}$.

Le monstre prometteur est donc cette hypothèse selon laquelle toutes les espèces pourraient virtuellement prendre origine dans un monstre. Doit-on encore, d'ailleurs, utiliser ce vocable: les monstres tératogènes restant, après tout, autant de formes normales en puissances.

Belle naïveté qui ne voit au plus loin qu'à l'horizon de la norme. Alors que, tout au contraire, prendre en compte la spécificité de l'être-là du monstre demande de penser la pluralité hors de ce retour au même. Mary douglas insiste sur ce fait que la souillure est toujours avant tout quelque chose qui n'est pas à sa place. Lui en attribuer une, c'est la résorber dans le sacré. C'est pourquoi cette vieille idée d'une loi aléatoire de la nature, que la tératologie se propose de dépasser, est en fait bien plus féconde. À partir d'une définition relativement stricte - le monstre est ce dissemblable d'avec ses géniteurs -, Aristote va décrire la production des monstres comme un phénomène faisant partie des lois de la nature : "Ce qui a conduit la nature à produire des monstres est le fait de ne pas engendrer des êtres semblables [aux géniteurs] par inachèvement ${ }^{34}$. » Le monstre est donc ici aussi conforme à la nature, mais cette fois-ci en ce qu'elle comporte, pour Aristote, d'aléatoire et d'indéterminé. Annick Jaulin développe sur ce problème dans son analyse de la pathologie politique chez cet auteur :

[...] on sait qu'avec la philosophie pratique on entre, pour Aristote, dans le domaine des choses qui peuvent toujours être autrement qu'elles ne sont, c'est-à-dire dans un domaine où l'aléatoire est la règle, de sorte que la dissemblance ne peut même plus être décrite comme monstruosité, mais seulement comme variation ${ }^{35}$.

Pour Aristote, la nature, parce qu'aléatoire, n'exclut pas les anomalies. C'est, bien sûr un point de vue normatif, puisque le monstre y répond des lois du système ; malgré cela, cette idée de variation admet a minima qu'à un genre donné réponde une pluralité de formes.

Nancy et Lacoue-Labarthe disaient le mélange constitutif du genre, pour expliquer que celui-ci soit, par essence, indéfini et illimité. La pensée aristotélicienne d'une production «naturelle » du monstre, bien qu'ouvrant le nombre des possibles, est encore en deçà de cette idée d'un bouleversement de la taxinomie normative. Les opérations de la fragmentation comme de l'interdisciplinarité constituent, en pratique et en théorie, un écart proprement inédit à toute pensée ou travail artistique les précédant : un écart par rapport à la notion même de genre. D'évidence, si l'inter remet en question la pratique disciplinaire, la définition de l'œuvre plasticienne et la grille de lecture critique s'y rapportant, c'est en ce qu'il se pose comme un espace de pensée à l'intérieur duquel on ne puisse pas attendre l'instauration d'un Ordre. Pierre Magnaud le rappelle dans son article Au-delà du spécisme, «[...] la leçon la plus vive du surgissement du monstrueux dans la nature et dans la société, c'est la remise en cause du fétichisme de l'identité $[. .]^{36}$. » Et il cite Montaigne, s'affranchissant de ce carcan identitaire en faisant d'un moi "ondoyant et divers» le "monstre au monde le plus exprès »: il y a, nous dit Montaigne, 
" autant de différence de nous à nous-mêmes que de nous à autrui ${ }^{37}$." C'est ce que démontrent avec brio les queer studies, notamment Judith Butler via son fameux Trouble dans le genre : l'identité genrée n'est ni stable, ni permanente. Par essence indéfinie et illimitée, disaient Lacoue-Labarthe et Nancy, ce qui est tout à fait adéquat dans cette perspective butlerienne selon laquelle l'infini des possibles du genre sexué doit se penser non pas dans la solidité d'une contre-culture, tout aussi standardisante, mais en dénaturalisant la norme de l'hétérosexualité obligatoire. Hors-genre et trans-genre ne sont pas pour Butler l'inverse du genre, comme le elle serait censé fonder l'alternative symétrique au il. Les identités genrées hors normes constituent, au contraire, l'alternative subversive aux normes hétéro et andro-centrée qui nous assujettissent: parce que les sujets sexuels ne répondent jamais parfaitement des injonctions normatives auxquelles ils-elles doivent se conformer, et que dans ce jeu de la norme ce jeu d'avec la norme, pour certains - se situe la possibilité de déconstruire les identités données. À l'encontre du concept de genre se faisant passer pour loi naturelle et immuable, Butler propose de penser une identité performative.

La performativité serait ce processus d'identifications réitérées au jour le jour, dans chaque acte, chaque parole, chaque prise de position, par lesquelles le moi se reconnaît comme moi. Par lesquelles, donc, tout un chacun peut subvertir la norme en réinventant sans cesse de nouvelles formations du sujet. Pour Butler, «Le genre est un phénomène complexe qui, en tant que totalité, est constamment différé, un idéal impossible à réaliser quel que soit le moment considérés8." Alors, plutôt que d'instaurer des identités sur une base contingente, c'est-à-dire en vue d'un objectif prédéterminé, elle propose à rebours que le genre - compris simultanément dans le sens politique de coalition - soit un «[...] assemblage ouvert permettant de multiples convergences et divergences sans qu'il soit nécessaire d'obéir à une finalité normative qui clôt les définitions ${ }^{39}$.»

Un genre pourrait exister en tant que tel sans donner à l'avance la forme idéale que devrait en adopter la structure. Nancy et Lacoue-Labarthe ne démentent pas ce trait dans leurs propos sur le romantisme : "On aura cependant remarqué, disent-ils, [...], qu'un pareil Witz ne cesse pas d'errer - sinon d'opérer - dans ce dispositif, entre système et chaos, entre les deux pôles de l'organon ${ }^{40}$.» Et d'insister sur cette hésitation caractéristique du "flou romantique", qu'ils qualifient comme jeu d'un écart à l'idéalisme et à la métaphysique qui s'y parachève, et qui, finalement, «[...] pourrait bien aussi parfois signifier que le romantisme détient par constitution une certaine impossibilité d'accommoder exactement la vision de l'idée ${ }^{41}$. "

31 L'unité obligatoire n'est l'apanage de rien que de la catégorie. Que Lacoue-Labarthe et Nancy fassent ici allusion au rapport signifiant-signifié, ils posent le problème d'un soidisant idéal de concordance de l'un à l'autre. Dans les arts plastiques, la gestalt a édifié cette illusion que la vision synchrone du fond et de la forme révélait le sens de l'œuvre, sens lui-même résoluble, unifiable, univoque : un sens, et même plus, un sens immédiat. Si la poésie romantique génère ce flou, si la forme fragmentaire ne permet pas d' accommoder exactement la vision de l'idée, c'est parce que, note Maurice Blanchot, en "Commençant de se rendre manifeste à elle-même grâce à la déclaration romantique, la littérature va désormais porter en elle cette question - la discontinuité ou la différence comme forme $[. . .]^{42}$ ». C'est l'exacte hypothèse - rapportée cette fois aux arts plastiques - qu'Yve-Alain Bois et Rosalind Krauss vont approfondir dans L'informe, mode d'emploi. Partant du constat d'un modernisme relevant avant tout d'une entreprise 
ontologique, Yve-Alain Bois en note les postulats dans une démonstration en quatre temps ${ }^{43}$ :

1 - En tout premier, l'art, particulièrement la peinture, s'adresse à un sens et un seul, la vision. Un postulat que l'on retrouve aux origines de mouvements artistiques (citons l'impressionnisme, comme peinture rétinienne), mais aussi dans les premiers écrits d'Hildebrand, de Fiedler, qui influeront sur ceux de Wölfflin, c'est-à-dire dans les fondements scientifiques de l'histoire de l'art comme discipline.

2 - Cette idée d'une pure vision implique une exclusion qui remonte au Laocoon de Lessing - à cette position dévalorisée, dans la hiérarchie qu'il propose, des arts du temps et des arts de l'espace : il y aurait une temporalité propre au visuel, qui est de l'ordre de l'immédiateté - pour Yve-Alain Bois, alors, «[...] le tableau se donne en un instant et ne s'adresse qu'à l'œil du spectateur ${ }^{44}$. »

3 - Bois qui s'appuie ensuite sur deux textes de Freud, Trois essais sur la théorie de la sexualité et Malaise dans la civilisation, pour démontrer comment cet art de la pure vision implique un face à face fondamental avec l'œuvre, et maintient de ce fait le corps du sujet percevant dans sa position érigée, loin de celle, horizontale, propre à l'animal. Et cela signifiant, selon Bois, que " Même s'il ne se veut plus une "fenêtre ouverte sur le monde", le tableau est une coupe verticale qui postule du spectateur l'oubli qu'il a les pieds dans la poussière ${ }^{45}$. " Contre le très pertinent «ni haut, ni bas » posé par Denis Hollier en préface à Documents, ici, toujours, cette même construction antithétique réduisant aux deux extrêmes, sacré et souillure.

4 - Pour conséquence, nous dit Bois «[...] l'art est une activité sublimatoire, qui rassemble (tout en le séparant de son corps) le sujet percevant. Il postule une activité synthétisante : l'artiste est censé concevoir l'œuvre comme totalité fermée [...], et la jouissance esthétique est indexée à cette plénitude formelle ${ }^{46}$. "

Voici résumés les mythes fondateurs du modernisme et de sa grille de lecture, gestalt fondée sur le rapport figure-fond: autant d'efforts associés qui, finalement, s'ils accommodent la vision, ne peuvent qu'empêcher que le genre ne se forme et ne se définisse par sa propre dynamique. Il n'y a pas loin de la pure vision à la pure pensée. Pour penser le genre comme lieu possible de contradiction de la norme, pour penser une multiplication infinie des catégories qui viendrait à bout du concept même, il faut suivre Butler et revenir à ce fondamental foucaldien: toujours, le fait de se donner pour fin absolue "l'unité " revient à la considérer comme condition préalable. Et à exclure tout le reste dans l'indéterminé, ce qui n'a plus d'identité - donc ne peut plus nuire -, la saleté qui jonche le sol, à nos pieds. Loin. En bas. Hors du cône de vision moderniste.

C'est la conclusion impérative d'Yve-Alain Bois: pour le modernisme un seul mot d'ordre, «Exclusion de tout ce qui se délite : l'œuvre doit avoir un début et une fin, tout désordre apparent se résorbant dans le fait qu'elle est limitée ${ }^{47}$. »

C'est peut-être aussi la limite de la couronne de fragments, cette idée qu'il faut encore une forme, une figure qui se détache sur un fond, pour que quelque chose soit visible, et que ce quelque chose fasse sens. Encore qu'avec le flou romantique, le dessin puisse en être plus riche que ce que l'image mentale suppose. Il y a ces jeux optiques des cartes d'Épinal, où l'on lit le profil d'une vieille dame sous son châle, et parce qu'on se concentre sur un pli du bonnet, soudain c'est le portrait d'une demoiselle en habit qui s'impose. Que l'on essaie de retrouver la vieille femme, la demoiselle redevient aussitôt 
insaisissable. Que l'on tente de voir les deux, simultanément, du coin de l'œil, et tout se brouille dans les premiers élancements d'une bonne migraine. Cette différence comme forme que note Blanchot ${ }^{48}$ montre pareille faille pour le fragment. La couronne, totalité plurielle qui fonde l'œuvre fragmentaire en unité, n'est jamais aussi que la forme négative, hors-d'œuvre, de la somme des totalités singulières. Comment résoudre cet écart entre les unités et le pluriel ? Est-il seulement encore possible d'y considérer une limite, un ordre amorçant début ou fin dans l'infini circulaire? Si, comme le dit Blanchot, c'est la discontinuité, la différence, qui font forme, il faut alors penser en conséquence l'altération de la structure taxinomique et de la grille de lecture. Et entendre de nouveau cette question posée par Lyotard : «Comment, en général, ce qui est forme peut-il aussi être transgression? [...] Comment ce qui est déviation, dérogation, déconstruction, peut-il être en même temps forme ?99 $^{49}$

Dans ce fait même d'interroger : y a-t-il une bonne forme de la transgression?, Lyotard parodie le système d'opposition binaire qu'il propose de dépasser. Il s'agit, après étude du si célèbre cas d'un fantasme analysé par Freud - connu sous cette formule « un enfant est battu»- de comprendre quel rôle joue la forme dans le travail de l'inconscient. Pour ce faire, Lyotard marque la différence d'avec la grille structuraliste sur laquelle repose la gestalt : a contrario, la logique spatiale de l'inconscient ne répond ni de règles d'opposition, ni de cette exigence de toujours spécifier les différents objets par ressemblance ou différenciation. Freud notait que les stades distincts du fantasme étudié ne correspondaient pas à une succession, chacun suppléant l'autre, et encore moins à une évolution comprise comme progrès du sens. À l'inverse de la grille structuraliste, l'inconscient joue la simultanéité; "Au contraire, [comme le note Krauss] les significations de tous les stades y demeurent suspendues, sous la forme d'un "mais aussi" 50 .» Euvre, mais aussi hors-d'œuvre. En ajoutant encore, dans le cas particulier de l'inter, l'infini de la combinatoire à celui de chaque discipline. La réponse, alors, à cette bonne forme de la transgression repose sur l'éviction du principe structuraliste de non-contradiction, sur cette idée que plusieurs choses antinomiques puissent être, simultanément. Krauss résume cela : «L'inconscient ne se contente pas de solliciter la transformation de toute chose en son contraire, il maintient l'une et l'autre ensemble ${ }^{51}$.»

Lors, si l'inconscient ne respecte pas les écarts entre les objets, s'il ne maintient pas de rapports constants - comme les intervalles du langage discursif - entre ses différentes parties, c'est d'évidence que la transgression ne peut relever d'une forme telle que l'entend la gestalt. La forme de la transgression est nécessairement rupture d'une rythmique, dit Lyotard, donc une « mauvaise forme »:

En d'autres termes la forme à laquelle nous avons affaire avec le fantasme n'est pas une bonne forme. Elle est certes une forme dans laquelle le désir reste pris, la forme prise par la transgression; mais elle est aussi la transgression, au moins potentielle, de la forme ${ }^{52}$

41 Une mauvaise forme que cet espace inconscient, la matrice, qui transgresse les silences constitutifs du discours et les distances constitutives de la représentation. C'est dans cette propriété du figural de parvenir - comme l'écrit Lyotard - à bloquer ensemble ce qui n'est pas compossible, que peuvent être dépassées la pure vision synthétisante de la gestalt et les disciplines ontologiques du modernisme. Que Nancy et Lacoue-Labarthe notent cette caractéristique du romantisme de ne plus permettre d'accommoder exactement la vision de l'idée, que l'inter induise une production artistique et une lecture transversales, c'est cette même faillite des écarts de productions. La matrice dissimule la 
contradiction et détruit la différence ; par elle, figure et fond sont donnés ensemble et occupent simultanément un même espace: la matrice détruit donc aussi la forme. Krauss et Bois nuancent ce terme de "destruction » pour mieux expliciter la notion d'informe: "L'informe n'est cependant pas simplement un effacement de la forme: c'est une opération qui défait la forme $[. . .]^{53}$.» C'est ce processus qui, en art, génère la "mauvaise forme». Outre référence incontournable à l'espace matrice de Lyotard, Krauss et Bois reviennent incessamment sur l'utilisation première de ce terme telle que définie par Bataille : c'était cet autre mot qu'il avait choisit pour évoquer le processus de « déviance » :

[...] informe, désignant un déclassement dans tous les sens du terme : minant à la fois la séparation entre l'espace et le temps (battement), les systèmes d'arpentages de l'espace (horizontalisation, production du plus bas que bas), les qualifications de la matière (bas matérialisme) et l'ordre structural des systèmes (entropie) $)^{54}$.

Un déclassement, il faut le préciser, c'est à entendre comme une production du hors norme, $d u$ hors genre, $d u$ hors taxinomie. Comme la fragmentation ou l'interdisciplinarité, l'informe opère sur les produits d'écarts ces déplacements irréguliers dont Lyotard dit qu'ils peuvent suffirent à transformer le singulier en pluriel, ou l'ici en ailleurs. L'informe n'est pas seulement un effacement de la forme, pas seulement non plus cette chute de la forme et du sens dans la décadence, la décrépitude, l'entropie et l'oubli. Pour Didi-Hubermann, pourtant, l'informe peut être théorisé comme une condition rythmique de la forme. Il l'expose dans un ouvrage, La ressemblance informe, dont le titre même, tourné en un oxymoron, contraint l'informe à la définition simpliste d'une déformation. Retour au monstre. Selon lui, «L'informe qualifierait donc un certain pouvoir qu'on les formes elles-mêmes de se déformer toujours, de passer subitement du semblable au dissemblable. » Autant revenir au système d'opposition binaire de la grille structuraliste : passage subit de l'un à l'autre, mais plus cet à la fois de l'espace de l'inconscient. En rabattant ainsi la question de la mauvaise forme à un axe unique, à la seule alternative de l'alternance, du positif au négatif, Didi-Hubermann revient en deçà de toute déconstruction. Pour Rosalind Krauss :

Voilà le risque que l'on prend à vouloir à tout prix mesurer l'informe à la ressemblance ou à la dissemblance, au lieu de s'aviser que "ressembler à rien " n'est ni dissembler de quelque chose en particulier, ni ressembler à quelque chose qui se trouverait être rien ${ }^{55}$.

Et tout cela, chez Lynch? Ces réflexions sur le statut équivoque de l'œuvre fragmentaire et du dépassement du genre disciplinaire pourraient être illustrées - avec ce manque d'inter, pour ne pas dire d'esprit, que dévoile trop souvent l'adjonction redondante - donc inepte - d'un visuel à un textuel dont on présuppose par là le sens prépondérant - l'un et l'autre toujours considérés par cet écart et seulement celui-ci de leurs formes respectives. Cela serait aisé, et si déplacé de développer le propos sur la thématique du monstre dans les différentes productions de Lynch. Et des monstres, il y en a. Pléthore. Mais rien d'utile à se laisser aller au piège de la ressemblance. Canguilhem le démontre dans Le normal et le pathologique, il faut soigneusement éviter tout rapprochement erroné entre anomalie et anomal, au risque d'abaisser toute solution de déclassement à une irrégularité de fait, et de produire encore une autre théorie normative des déviations.

En faisant œuvre fragmentaire, le Romantisme a soumit à sa critique le canon du genre et celui de la perfection esthétique. Le postulat de cette étude étant que 
l'interdisciplinarité propose pareille alternative quant aux pratiques et lectures essentialistes qui tendent à réduire l'art aux disciplines, et celles-ci en normes esthétiques. Pour ce faire, redisons-le, la question d'une prétendue norme de beauté, un idéal auquel serait opposé le monstre comme mauvaise forme, doit impérativement être dépassée. Kant, déjà, dans la Critique de la faculté de juger, invalidait le canon antique. L'idée d'un critère de perfection formelle relève en fait selon lui d'un objet imaginaire, un tracé contingent déterminé par la moyenne qui se dégage de la superposition d'un grand nombre d'image. Bataille pense de la même façon une beauté statistique contre l'idéal d'une perfection de la forme. Son essai Les écarts de la nature exemplifie le travail photographique de Francis Galton : à la fin du XIXe siècle, celui-ci superpose des séries de clichés de visages, ou bien de profils frappés sur des pièces de monnaies romaines, le résultat de ce travail composite montrant pour moyenne une forme unique qui, selon Bataille, n'aurait rien à envier à la beauté des sculptures antiques - il cite l'Hermès de Praxitèle. Bataille généralise le concept :

Si l'on photographie un nombre considérable de cailloux de dimensions semblables, mais de formes différentes, il est possible d'obtenir autre chose qu'une sphère, c'est-à-dire une figure géométrique. Il suffit de constater qu'une commune mesure approche nécessairement de la régularité des figures géométriques ${ }^{56}$.

Ainsi, la moyenne des images fixe sous nos yeux l'idéal du genre homogénéifié - mais c'est une opération de ce même genre de la génération propre au fragment, en cela qu'elle génère aussi ses propres discordances. La moyenne idéale est une synthèse de l'hétéroclite; elle n'est que l'illusion homogène d'une proposition composite, et n'est modèle que dans ce sens de spécimen, le type à partir duquel chaque être, chaque chose ne peut être que variation. C'est l'Idée-norme, chez Kant, allgemeinen Richtmass, sorte d'étalon universel qui définit l'espèce, à comparaison duquel chaque individu sera une réalisation variable et plus ou moins déviante. Jacques Darriulat résume les implications de cette notion:

[...] la proportion de l'Idéal ou la norme de la beauté, réalisée de façon indépassable dans la statuaire de la Grèce ancienne, n'est que le degré zéro de la représentation, un schème simplifié qui élimine les aberrations individuelles, une normalisation imaginaire des formes vivantes ${ }^{57}$.

Pour Bataille, pareillement, chaque forme individuelle échappe à cette commune mesure, et à quelque degré, est un monstre, un écart dont la nature est incontestablement responsable.

C'est pourquoi il ne faut pas parler des monstres, chez Lynch, mais bien du monstrueux. Pour lire et comprendre comment opère la déconstruction à l'œuvre dans la discipline même, dans cet idéal moderniste d'un corps intègre auquel rien ne manque, d'un tout unifié ; pour saisir, dit Krauss :

La production inévitable du monstrueux ou de l'hétérogène, par le procès même qui est destiné à exclure le non-généralisable, telle est la force qui crée la différence non logique à partir des catégories qui ont pour fin d'administrer logiquement la différence ${ }^{58}$.

Le monstrueux, chez Lynch, ne prend pas corps dans l'homme éléphant, le manchot, le nain, les collections de mouches mortes, l'oreille coupée encore sanguinolente. Certains polémiquent encore sur l'utilisation d'une tête d'agneau écorchée pour la fabrication du bébé de Eraserhead, ce qui revient peu ou prou à en soumettre la pertinence à cette ressemblance de l'homme à la bête qui conduit la physiognomonie de Lavater. Dans ce cas précis, le monstrueux ne réside pas dans l'utilisation d'une matière pour une autre ; le déplacement des chairs à vif d'un animal à la peau rose, lisse et tendre d'un bébé 
n'est rien de plus qu'abject, c'est-à-dire un retour à l'asservissement au thème normatif de ce qui est laid. Ce qui importe, ce qui est monstrueux dans la mise en forme de ce corps de nourrisson, c'est que justement, il ne ressemble à rien, et surtout pas à ce qu'il devrait être ${ }^{59}$. C'est toute la différence entre sacré et souillure, anomalie et anomal, entre l'informel et l'informe, dont Bataille voit l'issue dans l'idée de bas matérialisme, un matérialisme n'impliquant pas d'ontologie, n'impliquant pas que la matière est en soi. Il faudra revenir là-dessus : développer sur la symbolique de l'absence de forme telle que la présente Douglas, sur la sublimation de la matière chez Freud, et bien sûr relire la critique du schème hylémorphique que propose Simondon pour invoquer, contre l'idée de leur éduction, le concept d'une émergence des formes en relation avec leur milieu associé.

Où situer alors, dans l'œuvre de Lynch, non plus ce monstre que l'on montre, monstrare, mais celui qui montre, monere, ce qui indexe cette possibilité de défaire la discipline comme genre idéal et permanent, et de valider une lecture interdisciplinaire ? Dans la différence de l'espace inconscient, dans la matrice, «[...] en ce qu'elle réside [dit Lytoard] dans un espace qui est encore au-delà de l'intelligible ${ }^{60}$. » Il faut penser chez Lynch cet au-delà de l'espace isotrope et structuraliste de la gestalt. Si l'inter est une sorte de tissu conjonctif de l'unité plurielle, s'il permet de penser des coupes transversales comme autant de liens connexes, il pourrait fonctionner comme un bloc, ce morceau de papier froissé par lequel Lyotard illustre l'espace de l'inconscient. L'inter agirait alors comme par condensation, reconfigurant le champ spatial en réunissant certains éléments et leur permettant d'être de nouveau interprétés. Disons plus justement que cette spatialisation répondrait autant de l'idée de suspens, parce qu'il n'y a pas de déplacements au sens de Freud dans l'œuvre interdisciplinaire, ni à y trouver un quelconque fantasme définitoire, mais bien à établir une topologie qui, entre la condensation et l'accrochage, permette à la fois monimentum et monumentum, garde de l'esprit et esprit de la garde, c'est-à-dire de réactiver le caractère polysémique de l'œuvre d'art - ce que Nancy et Lacoue-Labarthe notent également dans l'idée d'un inachèvement critique propre au genre romantique.

La matrice permet cette mauvaise forme, et comme le rappelle Rosalind Krauss :

[...] cette forme-qui-est-aussi-transgression-de-la-forme est donnée précisément par l'action de la figure matricielle de Freud : c'est l'action de battre, qui est la pulsation du plaisir, mais aussi la pulsation de la mort [...]. Le battement est donc la forme de la récurrence, de la répétition, la forme par quoi se délite la forme, par où le temporel s'insinue au cœur du figural et l'altère en mauvaise forme, en informe ${ }^{61}$.

Ils sont nombreux, ces battements, chez Lynch, qui minent le travail de la structure. Il faudrait développer sur la figure - ne doit-on pas le dire au pluriel, les figures - de la femme clivée, qu'il emprunte à Bunuel: et sur l'inquiétante étrangeté que Freud accorde à l'automate, ce cas de redoublement dénonçant la relation de ressemblance de la copie au modèle. Cette structure de l'unheimliche, qui produit un double inquiétant, on la retrouve aussi dans le principe de répétition de cette scène, dans Mulholland Drive: cette première dispute amoureuse entre Rita et Betty - feinte, puisqu'il s'agit de répéter un script - qui sera de nouveau jouée par Betty quelques minutes plus tard, dans un tout autre registre, et avec un homme cette fois, un acteur, face au staff présent pour l'audition. Ce redoublement provoquant une perte totale de repères: chaque interprétation invalidant la justesse de l'autre, toutes deux dénonçant le déjà $\mathrm{vu}$, ces ressorts de chaque genre cinématographique. 

fiction, comme plus tard dans le même film cette scène au Silencio pendant laquelle une chanteuse, sur scène, s'écroule, alors que la bande son continue et que Monsieur Loyal répète : it's all recorded, tout est enregistré. L'abîme de la répétition - on rejoue le bout d'essai, on fait de nouveau semblant de faire semblant - fragmente le point de vue du spectateur et bloque dans la convulsion toute cohérence de sens. Le continuum du film, l'enchaînement, ne se donne plus comme flux, ni unité. Et la pure vision ne peut momentanément plus rassembler le corps du sujet percevant. C'est, en conséquence, un échec de la temporalité définitoire du médium comme tout unifié; ce hoquet provoque la désublimation du processus cinématographique en tant que flux, c'est-à-dire l'exaltation de la coupe et du montage. scènes de genre, de ce genre qui s'inscrit dans la pulsation de l'éros, mais tout autant, en écho au désir, de cet autre, lié au thanatos et à l'inéluctabilité de la revenance. En gardant au montage les deux prises, Lynch évince la forme idéale dans la compulsion de mort.

Dans l'incongru de cette répétition, la parodie touche ${ }^{62}$ : pour appuyer qu'il ne s'agit jamais que de cela, jouer, prétendre, édifier de toute pièce quelque chose qu'il suffirait, comme le disait Duchamp, de répéter suffisamment pour que cela devienne un goût - et fasse genre. Ici, l'ambivalence d'une interprétation à l'autre montrant bien, dans le battement du rire aux larmes, que ce goût oscille toujours dangereusement entre éros et thanatos : quand l'enfant est battu, tout prend place dans ce sens trouble du contact répété sur le corps, en ce qu'il peut avoir d'érotisant ou bien tenir de la maltraitance.

55 La force de l'œuvre interdisciplinaire de Lynch est de répéter les clichés par des chaînes métonymiques au travers de plusieurs disciplines, ces transpositions et répétitions en chargeant d'autant le sens - et le déconstruisant. Dans cet inexorable retour au même, le contenu cède inévitablement à l'entropie: au pire, l'angoisse provoque alors le délitement dans le double morbide; au mieux c'est le simulacre qui demeure, vide de sens, et finit par n'être plus qu'un bruit. Ce bruit que la théorie de l'information désigne comme tout ce qui est probable, cliché, inutile, et par là même nuisible au contenu. La répétition devient alors cette reconnaissance du même, mais du même comme rien. Et l'insignifiance réclame de revoir la structure.

56 L'interdisciplinarité ne fait pas système, ce n'est pas un principe d'assimilation totale, normatif, donc, et tout entier au service du sens comme autorité. L'indexer au bloc, c'est proposer qu'en elle le mouvement transversal de lecture, comme un suspens, puisse autant être permutation et proposition. Propositions sans cesse réitérées pour la garde de l'esprit, et en cela il faudra se détacher de la notion du fragment comme œuvre in progress que proposent Lacoue-Labarthe et Nancy : ce progrès annonçant la visée bien trop restrictive d'un état supposé final, aboutit, accomplit, et reléguant l'inachevé au rang d'état, dans le sens que lui donne les taille-douciers.

Le sens et l'être, la vie organique, sont instables : la suite devra donc être cherchée dans le principe de métastabilité, chez Simondon par exemple. On peut toutefois avancer, déjà, que l'interdisciplinarité doit permettre cette relation de la relativité de toute chose, destinée à résister à tout retranchement vers l'illustration ou dans la thématique: par l'interdisciplinarité, l'art doit être ce concept qui s'informe. Sans espace de l'un à l'autre. Tout comme Lyotard décrit que s'informe le corps érotique dans l'espace de l'inconscient : 
[...] le corps érotique, qui n'est pas le corps vécu érotiquement, mais la surface où s'inscrivent les localisations du désir, est le contraire d'un monde, au moins chez l'enfant, le pervers, l'hystérique : puzzle de régions, où la charge-décharge de la jouissance trouve ses lieux d'élection, mais puzzle que personne ni rien ne tient sous son œil pour en faire l'unité d'un tableau, et où chaque zone peut elle-même accepter plusieurs significations simultanées quant au plaisir ${ }^{63}$.

\section{BIBLIOGRAPHIE}

Bataille Georges, Les écarts de la nature, in Euvres complètes, t. I, Paris, La Pléiade, 1998.

Bois Yve-Alain, Krauss Rosalind, L'informe, mode d'emploi, Paris, Centre Georges Pompidou, 1996.

Butler Judith, Trouble dans le genre, pour un féminisme de la subversion, Paris, La Découverte, 2005.

Chion Michel, David Lynch, Paris, Éditions de l'Étoile/Cahier du cinéma, 2001.

Darriulat Jacques, « Le monstre et l'idéal », in Qu'est-ce qu'un monstre?, Annie Ibrahim (dir.), Paris, PUF, 2005.

Douglas Mary, De la souillure, essai sur les notions de pollution et de tabou, Paris, La Découverte, 2005.

Gayon Jean, « Les monstres prometteurs : évolution et tératologie », in Qu'est-ce qu'un monstre?, Annie Ibrahim (dir.), Paris, PUF, 2005.

Jaulin Annick, « Aristote et la pathologie politique », in Qu'est-ce qu'un monstre ?, Annie Ibrahim (dir.), Paris, PUF, 2005.

Lacoue-Labarthe Philippe, Nancy Jean-Luc, L'absolu littéraire, théorie de la littérature du romantisme allemand, Paris, Seuil, 1978.

Loubier Patrice, Du moderne au contemporain, deux versions de l'interdisciplinarité, in Penser l'indiscipline, Lynn Hughes et Marie-Josée Lafortune (dir.), Montréal, Optica, 2001.

Lyotard Jean-François, Discours, figure, Paris, Klincksieck, 2002.

Magnaud Pierre, «Au-delà du spécisme », in Qu'est-ce qu'un monstre ?, Annie Ibrahim (dir.), Paris, PUF, 2005.

\section{NOTES}

1. Michel Chion, David Lynch, Paris, Éditions de l'Étoile/Cahier du cinéma, 2001, p. 193.

2. Philippe Lacoue-Labarthe, Jean-Luc Nancy, L'absolu littéraire, théorie de la littérature du romantisme allemand, Paris, Seuil, 1978, p. 63.

3. Édité dans L'absolu littéraire, théorie de la littérature du romantisme allemand, Philippe LacoueLabarthe et Jean-Luc Nancy, Paris, Seuil, 1978, p. 126.

4. Philippe Lacoue-Labarthe, Jean-Luc Nancy, L'absolu littéraire, théorie de la littérature du romantisme allemand, Paris, Seuil, 1978, p. 63.

5. Patrice Loubier, Du moderne au contemporain, deux versions de l'interdisciplinarité, in Penser l'indiscipline, Lynn Hughes et Marie-Josée Lafortune (dir.), Montréal, Optica, 2001, p. 25. 
6. Philippe Lacoue-Labarthe, Jean-Luc Nancy, L'absolu littéraire, théorie de la littérature du romantisme allemand, Paris, Seuil, 1978, p. 64.

7. Ibid., p. 64.

8. Ibid., p. 64.

9. Ibid., p. 64. Comme indiqué entre parenthèses à la fin de la citation, le passage cite lui-même le fragment 82 de l'Athénaum, publié dans ce même ouvrage, p. 107.

10. Ibid., p. 69.

11. Patrice Loubier, Du moderne au contemporain, deux versions de l'interdisciplinarité, in Penser l'indiscipline, Lynn Hughes et Marie-Josée Lafortune (dir.), Montréal, Optica, 2001, p. 24.

12. Philippe Lacoue-Labarthe, Jean-Luc Nancy, L'absolu littéraire, théorie de la littérature du romantisme allemand, Paris, Seuil, 1978, p. 71.

13. Ibid., p. 67.

14. Ibid., p. 68.

15. Ibid., p. 71.

16. Ibid., note de bas de page p. 70 .

17. Ibid., p. 70.

18. Ibid., p. 376.

19. Cité par Philippe Lacoue-Labarthe et Jean-Luc Nancy, in L'absolu littéraire, théorie de la littérature du romantisme allemand, Paris, Seuil, 1978, p. 377.

20. Fragment 116 de l'Athenaum, reproduit par Lacoue-Labarthe et Jean-Luc Nancy, in L'absolu littéraire, théorie de la littérature du romantisme allemand, Paris, Seuil, 1978, p. 112.

21. Ibid.

22. Philippe Lacoue-Labarthe, Jean-Luc Nancy, L'absolu littéraire, théorie de la littérature du romantisme allemand, Paris, Seuil, 1978, p. 276.

23. Mary Douglas, De la souillure, essai sur les notions de pollution et de tabou, Paris, La Découverte, 2005 , p. 61.

24. Ibid., p. 61-62.

25. Ibid., p. 70.

26. Ibid., p. 73.

27. Ibid., p. 73.

28. Mary Douglas, De la souillure, essai sur les notions de pollution et de tabou, Paris, La Découverte, 2005, p. 72.

29. Ibid.

30. Jean Gayon, "Les monstres prometteurs: évolution et tératologie ", in Qu'est-ce qu'un monstre?, ouvrage collectif coordonné par Annie Ibrahim, Paris, PUF, 2005, p. 121.

31. Ibid., p. 121.

32. Ibid., p. 119.

33. Ibid., p. 119-120.

34. Cité par Annick Jaulin, dans l'article "Aristote et la pathologie politique ", in Qu'est-ce qu'un monstre ?, ouvrage collectif coordonné par Annie Ibrahim, Paris, PUF, 2005, p. 33.

35. Annick Jaulin, «Aristote et la pathologie politique », in Qu'est-ce qu'un monstre?, ouvrage collectif coordonné par Annie Ibrahim, Paris, PUF, 2005, p. 35.

36. Pierre Magnaud, "Au-delà du spécisme", in Qu'est-ce qu'un monstre?, ouvrage collectif coordonné par Annie Ibrahim, Paris, PUF, 2005, p. 60.

37. Cité par Pierre Magnaud, dans son article "Au-delà du spécisme ", in Qu'est-ce qu'un monstre?, ouvrage collectif coordonné par Annie Ibrahim, Paris, PUF, 2005, p. 60.

38. Judith Butler, Trouble dans le genre, pour un féminisme de la subversion, Paris, La Découverte, 2005, p. 83.

39. Ibid. 
40. Philippe Lacoue-Labarthe, Jean-Luc Nancy, L'absolu littéraire, théorie de la littérature du romantisme allemand, Paris, Seuil, 1978, p. 420.

41. Ibid.

42. Cité par Philippe Lacoue-Labarthe et Jean-Luc Nancy, in L'absolu littéraire, théorie de la littérature du romantisme allemand, Paris, Seuil, 1978, p. 421.

43. Yve-Alain Bois, Rosalind Krauss, L'informe, mode d'emploi, Paris, Centre Georges Pompidou, 1996, p. 16. Yve-Alain Bois précise d'ailleurs que, dans ces débuts, «Le "tactile" dont parle l'histoire de l'art n'est que la représentation visuelle du tactile : la matière n'y existe qu'informée, que mise en forme. »

44. Yve-Alain Bois, Rosalind Krauss, L'informe, mode d'emploi, Paris, Centre Georges Pompidou, 1996, p. 22.

45. Ibid.

46. Ibid.

47. Ibid.

48. Maurice Blanchot parle aussi de discontinuité : ceci convoque une autre opération de l'informe, le battement - voir le catalogue écrit par Yve-Alain Bois et Rosalind Krauss, L'informe, mode d'emploi, Paris, Centre Georges Pompidou, 1996, plus précisément p. 124-159.

49. Jean-François Lyotard, Discours, figure, Paris, Klincksieck, 2002, p. 349.

50. Yve-Alain Bois, Rosalind Krauss, L'informe, mode d'emploi, Paris, Centre Georges Pompidou, 1996, p. 97.

51. Ibid.

52. Jean-François Lyotard, Discours, figure, p. 350.

53. Yve-Alain Bois, Rosalind Krauss, L'informe, mode d'emploi, Paris, Centre Georges Pompidou, 1996, p. 99.

54. Ibid., p. 242.

55. Ibid., p. 74.

56. Georges Bataille, Les écarts de la nature, in CEuvres complètes, t. I, Paris, La Pléiade, 1998, p. 229.

57. Jacques Darriulat, «Le monstre et l'idéal », in Qu'est-ce qu'un monstre?, ouvrage collectif coordonné par Annie Ibrahim, Paris, PUF, 2005, p. 94.

58. Yve-Alain Bois, Rosalind Krauss, L'informe, mode d'emploi, Paris, Centre Georges Pompidou, 1996, p. 242.

59. Si l'on veut revenir à l'étude que propose Michel Chion du décalage toujours productif entre bande son et image chez Lynch, il faudrait rapprocher cette impression que provoque la mise en forme du bébé de l'écart tout aussi hérissant que produisent les piaillements aigus de gallinacés accompagnant la tétée des chiots, lors du dîner en famille, toujours dans Eraserhead.

60. Jean-François Lyotard, Discours, figure, p. 338.

61. Yve-Alain Bois, Rosalind Krauss, L'informe, mode d'emploi, Paris, Centre Georges Pompidou, 1996, p. 99.

62. Autre œuvre pour confirmation : la série des Rabbits par exemple, faux semblant grotesque de tout ce que le cinéma, ou les séries télévisées, peuvent véhiculer comme convenances et idées reçues. Le décor est parfait d'ineptie. Les rires préenregistrés agacent juste ce qu'il faut. Mais là où la parodie touche, c'est dans l'incongru de la personnification des lapins grandeur nature : quand le générique donne le nom des acteurs présents sous les masques.

63. Jean-François Lyotard, Discours, figure, p. 338. 


\section{RÉSUMÉS}

Comparer la discipline au fragment, pour l'étude de l'œuvre de David Lynch, c'est poser les enjeux de cet interdisciplinaire caractéristique de la postmodernité : parce que la fragmentation, comme l'inter, induit cette relativité qui se joue de la catégorie de genre, et détache enfin le concept de monstre d'une relation binaire d'avec toute taxinomie.

\section{INDEX}

Personnes citées : Butler (Judith), Krauss (Rosalind), Bois (Yve-Alain), Nancy (Jean-Luc), LacoueLabarthe (Philippe), Douglas (Mary), Lynch (David)

Mots-clés : classification, fragmentation, genre, interdisciplinarité, monstre, monstrueux, norme

\section{AUTEUR}

\section{MARION DELAGE DE LUGET}

Doctorante en philosophie esthétique, professeur d'histoire et de sémiologie de l'art, ainsi que critique. 